\title{
Análise por envoltória de dados utilizada para medir o desempenho relativo da cobrança pelo uso da água nas bacias hidrográficas do estado do Ceará
}

\author{
Data envelopment analysis used to measure the relative performance \\ of charging for water use in the river basins of the state of Ceará
}

\section{Marcus Vinícius Sousa Rodrigues \\ Marisete Dantas de Aquino Antônio Clécio Fontelles Thomaz}

\author{
Submetido: 16-10-14 \\ Revisão: 04-03-15 \\ Aprovado: 16-06-15
}

RESUMO: A cobrança pela água no Ceará teve início no ano 1996, adotando como base de cálculo apenas o consumo efetivo, não fazendo uma distinção entre captação e consumo. Sabe-se que múltiplos são os fatores que podem ser usados em uma análise de eficiência do instrumento de cobrançaem uma bacia hidrográfica. Para uma avaliação que considera simultaneamente vários fatores utiliza-se um modelo de análise multicritério. $\mathrm{O}$ objetivo deste trabalho consiste em avaliar o desempenho relativo, por meio da aplicação da análise por envoltória de dados, da cobrança nas principais categorias de usodas bacias do Estado. Foi aplicado, portanto, o modelo BCC (Banker, Charnes e Cooper), com orientação a produto, para o cálculo dos desempenhos relativos, com o auxílio da ferramenta computacional SIAD (Sistema Integrado de Apoio à Decisão). A análise feita mostrou que o setor industrial em todo o Estado se destaca dos demais setores, apresentando uma eficiência média de aproximadamente $92,6 \%$. Por outro lado o abastecimento e a irrigação se apresentam com baixas eficiências médias, iguais a aproximadamente $45,9 \%$ e 29,9\%, respectivamente. O modelo se mostrou eficaz no que fora proposto, ou seja, obter um diagnóstico em todo o Estado a respeito da eficiência da cobrança nas principais categorias de uso. Assim, recomenda-se fortemente o uso desta metodologia científica como apoio no processo de tomadas de decisōes no setor de gestáo de águas de modo a obter indicadores de desempenho desse instrumento.

PALAVRAS-CHAVES: Análise multicritério, Análise por envoltória de dados, Seleção de fatores.
ABSTRACT: In Ceará charging for water began in 1996, taking actual consumption alone as a basis for calculation, without distinguishing between capture and consumption. It is known that multiple factors can be used in an efficiency analysis of the collection instrument in a watershed. For an evaluation that simultaneously considers various factors, a multi-criteria analysis model is used. The main objective of this study is to evaluate the relative performance of the collection in the main categories of use of state basins by implementing Data Envelopment Analysis. Thus the BCC (Banker, Charnes "; Cooper) model was applied with product-based orientation for the calculation of the relative performances, with the help of the SIAD (Decision Support Integrated System) computational tool. The analysis showed that the industrial sector throughout the state stands out among sectors, with an average efficiency of approximately $92.6 \%$. On the other hand, supply and irrigation have a low average efficiency, equal to approximately $45.9 \%$ and $29.9 \%$, respectively. The model was effective as had been proposed, that is, it was able to obtain a diagnosis throughout the state regarding collection efficiency in the main categories of use. Hence, the use of this scientific methodology to support the decision-making process in the water management sector is strongly recommended in order to obtain performance indicators of that instrument.

KEY-WORDS: Multi-criteria analysis, Data Envelopment Analysis, Selection of variable.

\section{INTRODUÇÃO}

O modelo de gerenciamentos dos recursos hídricos no Brasil foi desenvolvido tendo como base a escassez e os conflitos provocados pelos vários usos das águas. Assim, a água foi definida como um bem natural, limitado e dotado de valor econômico.As políticas de recursos hídricos, tanto nacional como estaduais, 
adotaram basicamente instrumentos de gerenciamento, dentre os quais se destacam a outorga pelo direito do uso da água e a cobrança pelo uso da água.

O Estado do Ceará instituiu a sua Política de Estado de Recursos Hídricos (PERH), por meio da Lei no 11.996, de 24 de julho de 1992 . Nesta Lei, já se previa como instrumento de gestáo a cobrança pelo uso dos recursos hídricos. Posteriormente, ela foi substituída pela Lei no 14.844 , de 28 de dezembro de 2010. O Estado do Ceará foi o primeiro estado da união a cobrar efetivamente pelo uso de seus recursos hídricos.

A cobrança no Ceará foi efetivada por meio do Decreto $n^{\circ} 24.264$, de 12 de novembro de 1996, onde inicialmente foram cobradas as retiradas de água destinadas aos usos nas indústrias e nas concessionárias de serviço de água potável. Conforme Rodrigues e Aquino (2014), somente a partir de 2004 essa cobrança passa a ser aplicada para as demais finalidades de uso, a saber: indústria, abastecimento público, irrigação, aquicultura, água mineral e demais categorias.

A Companhia de Gestão dos Recursos Hídricos do Estado do Ceará (COGERH) é o órgão responsável pelo faturamento e pela arrecadação dos recursos financeiros oriundos dessa cobrança, atuando como uma espécie de agência para todas as bacias hidrográficas do Estado.

Os recursos hídricos no Estado do Ceará deverão ser cobrados de acordo com as peculiaridades de cada bacia, sendo previsto também a cobrança pelo lançamento de efluentes (CEARÁ, 2010). Entretanto, mesmo já havendo emissão de outorgas para o lançamento de cargas poluentes em corpos hídricos, essa categoria de cobrança ainda não está sendo praticada.

O modelo adotado atualmente para o cálculo da cobrança pela água bruta apresenta a seguinte expressão matemática

$$
\mathrm{T}(\mathrm{u})=\mathrm{T}_{\mathrm{ef}} \mathrm{V}_{\mathrm{ef}}
$$

em que: $\mathrm{T}(\mathrm{u})$ é a tarifa paga pelo usuário pela água bruta, em R\$;; $T_{\text {ef }}$ é a tarifa padrão de consumo, em $\mathrm{R} \$ / \mathrm{m}^{3} ; \mathrm{e}, \mathrm{V}_{\mathrm{ef}}$ é o volume efetivamente durante o mês pelo usuário, em $\mathrm{m}^{3}$.

A metodologia cearense de cobrança pela água pode ser considerada bem particular, uma vez que adota como base de cálculo apenas o consumo efetivo, não existindo uma distinção clara entre captação e consumo.

É importante ressaltar que tarifa de consumo, $\mathrm{T}_{\mathrm{ef}}$, varia de acordo como os seguintes critérios: tipo de uso; localização que se encontra o usuário (no abastecimento público); quem realiza a captação, se o próprio usuário ou COGERH; e conforme o volume consumido pelo usuário (na irrigação).

Segundo Aquino et al. (2013), o valor da tarifa e os critérios de cobrança são atualizados por meio de decreto do governador do Estado do Ceará. Atualmente, está em vigor o Decreto no 31.195, de 16 de abril de 2013, que dispóe sobre a cobrança pela água bruta, tanto superficial como subterrânea, do Estado ou da União por delegação de competência, além de fixar os valores das tarifas de consumo a serem cobradas nas bacias cearenses.

Por lei, a cobrança deve decorrer da outorga de direito de uso da água, emitida pela Secretaria de Estado de Recursos Hídricos (SRH). No entanto, na prática o que se vê é a existência de usuários faturados (que pagam pelo consumo de água) que não apresentam outorgas emitidas. Esse fato pode ser considerado como uma falha ou ineficiência do sistema de gerenciamento dos recursos hídricos do Estado.

A avaliação de desempenho entre unidades de atividade tais como bancos, hospitais, escolas, etc., é uma grande preocupação para o setor de administração, e o desenvolvimento de ferramentas para avaliar esse desempenho se expandiu consideravelmente nos últimos anos (ATHANASSOPOULOS, 2012).

Os índices e indicadores vêm sendo utilizados para avaliar a eficiência de planos e políticas públicas, e assim obter diagnósticos e direçóes a serem tomadas.Estes índices e indicadores podem ser pensados como fatores a serem considerados em uma análise para medir o desempenho (ou a eficiência) de determinada política pública, ou de algum instrumento específico dessa política.

Um indicador é formado por um conjunto de índices que fornece uma informação sobre uma dada realidade. Tanto indicador como índice pode ser elaborado para cumprir as funções de simplificação, quantificação, análise e comunicação, permitindo assim um melhor entendimento de fenômenos considerados complexos (CAMPOS et al., 2014).

Múltiplos são os critérios (ou fatores) que podem ser usados para se fazer uma análise da eficiência da cobrança entre as categorias de uso de uma bacia hidrográfica. Contudo, uma análise como essa requer o uso de uma metodologia científica chamada de análise multicritério.

A análise multicritério tem como finalidade atribuir uma nota sobre determinado objetivo a ser alcançado, por meio de um equacionamento no qual constam os critérios a serem considerados, os pesos 
atribuídos e um ordenamento entre eles. Dessa forma, as soluções obtidas podem ser ordenadas, de modo que seja possível selecionar a mais desejável ou ainda agrupar as melhores (TREVISAN et al., 2011).

A metodologia de análise multicritério surgiu como uma técnica de decisão nas décadas 1960 e 1970, como resultado das operaçóes de investigação que ocorreram durante a Segunda Guerra Mundial (HAJKOWICZ; COLLINS, 2007).

A técnica de análise multicritério adotada nesta pesquisa foi à análise por envoltória de dados (AED), também conhecida como DEA (sigla em inglês de Data Envelopment Analysis). Segundo Banaeian et al. (2011), essa técnica pode ser empregada a um conjunto de unidades de produçáo, comumente chamadas de unidades tomadoras de decisão (UTD), de modo a classificá-las de acordo com as suas performances.

Além da eficiência essa metodologiaé capaz de determinar metas a serem almejadas para unidades ineficientes. Uma UTD (também chamada de DMU, da sigla em inglês de decision making unit) pode representar qualquer conjunto de organizaçóes ou departamentos que realizam fundamentalmente a mesma tarefa com o mesmo conjunto de variáveis (ADLER; YAZHEMSKY, 2010).

O objetivo deste trabalho consiste em selecionar os fatores mais significativos de modo que sejam usados, por meio da aplicação da Análise por Envoltória de Dados, para avaliar o desempenho relativo do instrumento da cobrança pelo uso da água bruta das principais categorias de uso (indústria, abastecimento e irrigação) de todas as bacias hidrográficas do Estado do Ceará, obtendo assim um diagnóstico geral da eficiência desse instrumento.

\section{MATERIAL E MÉTODOS}

\section{Conceito de eficiência}

Em uma análise em que se objetiva avaliar o desempenho de um conjunto de UTDs, como se propóe neste trabalho, é de extrema importância saber o significado preciso dos seguintes termos: eficácia, produtividade e eficiência.

A eficácia se relaciona com o que é produzido, sem levar em conta os recursos empregados na produção. Logo, a eficácia pode ser definida como a capacidade que uma unidade produtora tem de alcançar uma meta de produção. Contudo, não basta uma unidade ser eficaz, ele deve ser também produtiva.

Em um processo produtivo os recursos (ou insumos) empregados devem ser utilizados da melhor forma possível de modo que não haja excessos. A medida de produtividade pode ser avaliada para comparar o desempenho entre várias unidades produtivas.

Matematicamente, a medida de produtividade total para $m$ insumos e $s$ produtos, é dada pela seguinte expressão:

Produtividade total $=\sum_{\mathrm{i}=1}^{\mathrm{s}} \mathrm{U}_{\mathrm{i}} \mathrm{Y}_{\mathrm{i}} / \sum_{\mathrm{j}=1}^{\mathrm{m}} \mathrm{V}_{\mathrm{j}} \mathrm{X}_{\mathrm{j}}$

onde: $\mathrm{U}_{\mathrm{i}}$ é o peso do i-ésimo produto, $\mathrm{Y}_{\mathrm{i}}(\mathrm{i}=1, \ldots, \mathrm{s})$; e, $V_{j}$ é o peso do j-ésimo insumo, $X_{j}(j=1, \ldots, m)$.

Os pesos podem ser atribuídos de acordo com a importância do produto ou do insumo, sendo normalmente escolhido de forma subjetiva pelos tomadores de decisão (RODRIGUES, 2014).

O conceito de produtividade, dado pela equação (2), pode ser usado para comparar várias unidades produtivas, sendo possível investigar a razão de uma unidade não ser tão produtiva quando comparada a outra.

O conceito de eficiência está relacionado à comparação de produtividade entre várias unidades, sendo, portanto uma medida relativa. A medida de eficiência de uma UTD pode ser obtida por meio de uma análise detalhada da relação existente entreinsumos e produtos. Esta relaçáo é denominada retornos de escala.

Dado um conjunto de insumos empregados na fabricação de produtos, então a função de produção define uma relaçáo ideal para a produção da quantidade máxima de produtos a partir de determinados insumos (EL-MAHGARY, 1995).

$\mathrm{Na}$ Figura 1 o eixo $x$ representa os insumos e o eixo y representa os produtos de uma determinada produção.A curva $S$ indica o máximo que foi produzido para cada nível de recurso, sendo chamada de Fronteira de Eficiência, enquanto a área OACEO abaixo da curva $S$ é chamada de Conjunto Viável de Produçáo e todo ponto localizado nessa região representa uma atividade produtiva.Um ponto sobre a curva $S$ indica a quantidade máxima de produtos para um dado nível de insumo, ou ainda a quantidade mínima de insumos necessária para atingir um nível estabelecido de produção. Assim, as unidades A e C são consideradas eficientes, entretanto a unidade A é mais produtiva que $\mathrm{C}$, pois a reta $\mathrm{OA}$ apresenta um coeficiente angular maior que a da reta OC. 


\section{Modelagens matemáticas de AED}

A AED é uma técnica de programação matemática que permite avaliar o grau de desempenho (ou eficiência) de um conjunto de UTDs semelhantes (apresentando o mesmo conjunto de insumos e de produtos), onde são considerados os recursos de que se dispóe com os produtos alcançados (BANKER, 1993).

Para Barbosa; Bastos (2014), a técnica de AED permite uma análise de eficiência comparativa de um conjunto de UTDs, demonstrando como uma unidade está operando em relação às demais do grupo, obtendo desse modo um diagnóstico completo de todo o conjunto.

Segundo Marjanovic et al. (2014), a abordagem AED para o cálculo da eficiência como uma técnica que não exige uma relação funcional, surgiu em contraste as abordagens tradicionais de estatística tais como a regressáo.

Um modelo de análise por envoltória de dados permite que seja feita uma avaliaçáo da eficiência de um conjunto de UTDs por meio da construçáo de uma fronteira linear por partes, chamada fronteira eficiente. As melhores relaçóes "produtos/insumos" são consideradas mais eficientes, estando situadas na fronteira eficiente, enquanto as menos eficientes estarão situadas na região abaixo dessa curva, denominada de envoltória convexa.

$\mathrm{Na}$ Figura 2 apresenta ilustrada uma fronteira de eficiência de um conjunto de UTDs, construída a partir das medidas de eficiência desse conjunto.

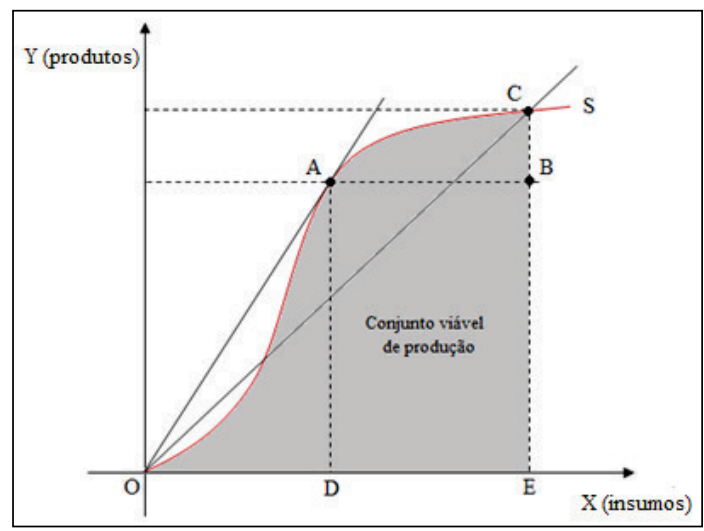

FIGURA 1. Curva de um processo produtivo.
Conforme a figura é possível identificar que as UTDs eficientes estão situadas nessa fronteira, enquanto as UTDs não eficientes estáo localizadas na região convexa, abaixo da fronteira de eficiência.

Segundo Silveira et al. (2012), existem dois modelos clássicos de AED: o modelo CCR (também conhecido como CRS, da sigla em inglês Constant Return to Scale), que admite retornos de escala constante, e modelo BCC (também conhecido como VRS, da sigla em inglês de Variable Return to Scale), que admite retornos variáveis de escala.

De acordo com Souza e Wilhelm (2009), as UTDs são comparadas conforme o conceito de eficiência de Farrel, que é a razão entre a soma ponderada das saídas (ou produtos) e a soma ponderada das entradas(ou insumos) de cada UTD.

É importante ressaltar o fato de que as eficiências medidas pelos modelos de análise por envoltória de dados se referem a uma eficiência relativa, e essa medida depende do modelo AED empregado, sendo determinada por três componentes: a superfície envoltória, a orientação e os pesos das variáveis (ALI et al., 1995).

A grande vantagem de se usar um modelo AED é que ele náo necessita a priori do conhecimento dos pesos (multiplicadores) dos insumos e dos produtos de cada UTD. Isto é, o próprio modelo é capaz de definir para cada UTD o conjunto de pesos para os seus fatores (insumos e produtos).

A única exigência é que todas as unidades avaliadas tenham uma medida de eficiência relativa igual ou

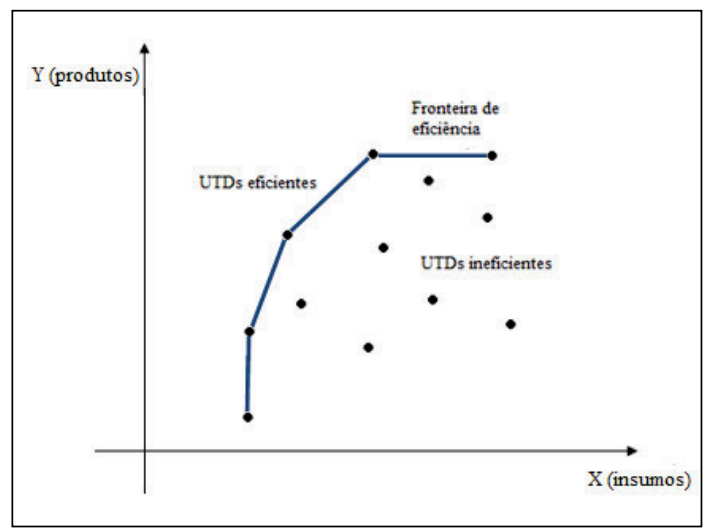

FIGURA 2. Fronteira de eficiência. 
menor do que a unidade. As unidades que apresentam uma eficiência igual a 1 são classificadas como eficientes, e caso contrário, são classificadas como ineficientes.

As unidades tidas como eficientes servem de referência para outras unidades consideradas ineficientes, sendo chamadas de benchmarks, de modo que essas unidades ineficientes estabeleçam metas a serem alcançadas com a finalidade de melhorarem as suas performances, aumentando assim as suas eficiências.

Logo, as unidades que se apresentam abaixo da fronteira de eficiência, tidas como ineficientes, devem ser projetadas em direção à fronteira eficiente, alcançado assim as suas parceiras eficientes. Uma UTD ineficiente pode atingir a fronteira eficiente de duas formas distintas:

a) Por meio da minimização das entradas (insumos), mantendo constantes as saídas (produtos), chamada de orientação a insumo;

b) Por meio da maximização das saídas (produtos), mantendo constantes as entradas (insumos), chamada de orientação a produto.

$\mathrm{Na}$ orientação a insumo, o principal objetivo de uma unidade ineficiente avaliada é ganhar eficiência, reduzindo o excesso de consumo de insumos, mantendo a produçáo constante. Enquanto na orientação a produto, o principal objetivo de uma unidade ineficiente avaliada é ganhar eficiência, aumentando a sua produçáo, mantendo o consumo de insumos constante.Em seguida, será feita uma descrição de cada um desses modelos de AED considerados clássicos, tanto com orientação a insumo como com orientação a produto.

\section{Modelo CCR}

A metodologia AED teve início com o trabalho publicado por Charnes; Cooper e Rhodes (1978) que avaliou a eficiência de programas escolares no Estado do Texas - USA, ficando conhecido como modelo CCR e sendo desenvolvido originalmente com uma orientação a insumo.

O modelo CCR trabalha com retornos constantes de escala. Isto é, qualquer variaçáo nos insumos implica em uma variaçáo proporcional nos produtos. A eficiência é determinada por meio da otimizaçáo da razão entre a soma ponderada dos produtos e a soma ponderada dos insumos. O cálculo da eficiência de um modelo AED pode usar tanto medidas orientadas a insumo como medidas orientadas a produto.
Suponha que existam $n$ UTDs, semelhantes entre si, utilizando $m$ insumos e $s$ produtos. Assim, em um modelo CCR, com orientação a insumo, os pesos dos insumos e dos produtos de uma unidade objeto, $\mathrm{UTD}_{\mathrm{O}}$, são determinados através da resolução do seguinte problema de programação matemática,

$$
\max E_{f O}=\sum_{i=1}^{s} U_{i} Y_{i O}
$$

Sujeito a:

$$
\begin{aligned}
& \sum_{j=1}^{m} V_{j} X_{j O}=1 \\
& \sum_{i=1}^{s} U_{i} Y_{i k}-\sum_{j=1}^{m} V_{j} X_{j k} \leq 0, \quad(k=1, \ldots, n) \\
& U_{i} \geq 0(i=1, \ldots, s) ; \quad V_{j} \geq 0(j=1, \ldots, m)
\end{aligned}
$$

onde: $\mathrm{E}_{\mathrm{fo}}$ é a eficiência relativa da $\mathrm{UTD}_{\mathrm{O}} ; \mathrm{Y}_{\mathrm{ik}} \mathrm{e}$ $\mathrm{X}_{\mathrm{jk}}$ são as quantidades de produto observado $\mathrm{i}$ da unidade $\mathrm{k}$ e de insumo observado $\mathrm{j}$ da unidade $\mathrm{k}$, respectivamente; $U_{\mathrm{i}} \mathrm{e} \mathrm{V}_{\mathrm{i}}$ são os pesos dados ao produto i e ao insumo j, respectivamente; e, $\mathrm{Y}_{\mathrm{io}}$ e $\mathrm{X}_{\mathrm{io}}$ são as quantidades do produto i e do insumo $\mathrm{j}$ da unidade objeto, respectivamente.

O problema de PML apresentado nas equaçóes (3) e (4) é comumente conhecida como Modelo dos Multiplicadores, também chamado de Primal, com orientação a insumo. $\mathrm{O}$ nome orientação a insumo vem do fato de que a eficiência é alcançada por intermédio da redução dos recursos.

Em seguida, serão apresentadas as formulaçóes do modelo CCR orientado a produto. Vale lembra que nesse tipo de orientação, as saídas (produtos) são maximizadas enquanto as entradas (insumos) permanecem inalteradas. Assim, a modelagem dos multiplicadores para o modelo CCR, orientado a produto, é dada da seguinte forma

$$
\sum_{i=1}^{s} U_{i} Y_{i k}-\sum_{j=1}^{m} V_{j} X_{j k} \leq 0
$$


Sujeito a:

$\sum_{\mathrm{i}=1}^{\mathrm{s}} \mathrm{U}_{\mathrm{i}} \mathrm{Y}_{\mathrm{iO}}=1$

$\sum_{i=1}^{s} U_{i} Y_{i k}-\sum_{j=1}^{m} V_{j} X_{j k} \leq 0$,

$(\mathrm{k}=1, \ldots, \mathrm{n})$

$U_{i} \geq 0(i=1, \ldots, s) ; V_{j} \geq 0(j=1, \ldots, m)$

Segundo Gomes et al. (2012), o modelo multiplicador trata da relação das somas ponderadas de produtos e insumos com os pesos escolhidos de modo a se tornar mais favorável a cada UTD analisada.É importante enfatizar que na modelagem AED dos multiplicadores, tanto com orientação a insumo como com orientação a produto, as incógnitas de decisão são os pesos $\mathrm{U}_{\mathrm{i}} \mathrm{eV}_{\mathrm{j}}$. Assim, pode-se afirmar que o conjunto dos pesos encontrados para cada UTD analisada deve ser tal que a medida da eficiência seja máxima.

\section{Modelo BCC}

Este modelo foi desenvolvido por Banker, Charnes e Cooper (1984), onde os retornos de escala são considerados variáveis, sendo conhecido como modelo BCC em homenagem aos autores.

O modelo passa a admitir tecnologias com retornos variáveis de escala, o que resulta em uma fronteira formada por combinaçóes convexas de unidades eficientes. De acordo com Gomes Júnior et al. (2013), este modelo adota o axioma da convexidade ao invés do axioma da proporcionalidade do modelo CCR.

A suposição de retornos constantes de escala do modelo CCR é relaxada para retornos de escala variáveis no modelo BCC, por intermédio da adição de uma variável livre, para orientação a insumo, e para orientação a produto.

Assim, o modelo BCC, orientado a insumo pode ser dado pelo problema de PML seguinte

$$
\max E_{f O}=\sum_{i=1}^{s} U_{i} Y_{i O}+U_{*}
$$

$$
\begin{aligned}
& \sum_{j=1}^{m} V_{j} X_{j O}=1 \\
& \sum_{i=1}^{s} U_{i} Y_{i k}-\sum_{j=1}^{m} V_{j} X_{j k}+U_{*} \leq 0, \quad(k=1, \ldots, n)
\end{aligned}
$$

$U_{i} \geq 0(i=1, \ldots, s) ; V_{j} \geq 0(j=1, \ldots, m) ; U_{*} \in \mathbb{R}$

Se a variável livre for positiva, então, o modelo apresenta rendimentos de escala náo decrescente (RND), enquanto se a variável for negativa, o modelo se apresenta com rendimentos de escala náo crescente (RNC).

A modelagem AED dos multiplicadores para o modelo BCC, orientado a produto, é dada da seguinte forma

$$
\min \mathrm{h}_{\mathrm{O}}=\sum_{\mathrm{j}=1}^{\mathrm{m}} \mathrm{V}_{\mathrm{j}} \mathrm{X}_{\mathrm{jO}}+\mathrm{V}_{*}
$$

Sujeito a:

$$
\begin{aligned}
& \sum_{i=1}^{s} U_{i} Y_{i O}=1 \\
& \sum_{i=1}^{s} U_{i} Y_{i k}-\sum_{j=1}^{m} V_{j} X_{j k}+V_{*} \leq 0, \quad(k=1, \ldots, n)
\end{aligned}
$$

$\mathrm{U}_{\mathrm{i}} \geq 0(\mathrm{i}=1, \ldots, \mathrm{s}) ; \mathrm{V}_{\mathrm{j}} \geq 0(\mathrm{j}=1, \ldots, \mathrm{m}) ; \mathrm{V}_{*} \in \mathbb{R}$

Se a variável livre for positiva, então, o modelo apresenta rendimentos de escala não crescente (RNC), enquanto se esta variável for negativa, o modelo se apresenta com rendimentos de escala não decrescente (RND).

\section{Unidades tomadoras de decisão (UTD)}

Uma metodologia AED para análise de eficiência de um conjunto de UTDs pode ser composta basicamente dos seguintes estágios:

1. Seleção das UTDs;

2. Definiçáo dos insumos e produtos (fatores de avaliação);

3. Escolha e aplicação do modelo;

4. Análise e interpretação dos resultados.

Sujeito a: 
Das categorias de uso de água bruta considerada pelo instrumento de cobrança, se destacam, tanto em relação ao consumo de água bruta e/ou faturamento com a cobrança, os setores da indústria, do abastecimento e da irrigação.

Assim, a pesquisa consistiu de uma análise do desempenho (eficiência) relativo do instrumento de cobrança pela água bruta dos principais usos considerados (indústria, abastecimento público, e irrigação) nas bacias cearenses, em um período de um ano (ou doze meses). Essa análise foi realizada por meio da aplicação da ferramenta de AED que tem como base um modelo clássico de produtividade com insumos e produtos.

Atualmente, o Estado apresenta-se dividido em doze bacias hidrográficas, a saber: Metropolitana, Litoral, Curu, Alto Jaguaribe, Médio Jaguaribe, Baixo Jaguaribe, Salgado, Banabuiú, Acaraú, Coreaú, Sertóes de Crateús e Serra da Ibiapaba. Entretanto, os dados obtidos para as bacias dos Sertóes de Crateús e da Serra da Ibiapaba ainda se encontram juntos como bacia do Parnaíba.

O conjunto analisado na pesquisa foi composto ao todo por 33 UTDs, listadas e identificadas no Quadro 1. Assim, cada UTD representa uma das categorias de uso (indústria, abastecimento público ou irrigação) de uma das onzes bacias hidrográficas do Estado consideradas.

Em seguida são apresentados os fatores de avaliaçáo utilizados para obter o modelo final de avaliaçáo.

\section{Conjunto de fatores para avaliação}

Os dados desta pesquisa são referentes aos instrumentos da cobrança pela água bruta e da outorga pelo direito de uso da água, sendo usadospara comporo conjunto de fatores que avaliaram o desempenho relativo da cobrança nos setores de uso da indústria, do abastecimento público e da irrigação nas bacias hidrográficas do Estado do Ceará.

A principal fonte de dados para esta pesquisa foi obtido junto à COGERH. Os dados fornecidos são referentes ao faturamento com a cobrança pelo uso da água bruta e às outorgas emitidas pela Secretaria de Estado dos Recursos Hídricos (SRH), em todas as bacias hidrográficas do Estado.

$\mathrm{O}$ relatório de cobrança, fornecido pela $\mathrm{CO}$ GERH, refere-se a um período de 12 meses (um ano) que se inicia em maio de 2012 e vai até o abril de 2013. Enquanto, os relatórios de outorgas emitidas, tanto para águas superficiais quanto para águas subterrâneas, referem-se até o mês de abril de 2013.

QUADRO 1

Unidades tomadoras de decisão avaliadas na pesquisa

\begin{tabular}{|l|l|l|}
\hline \multicolumn{1}{|c|}{ Indústria } & \multicolumn{1}{|c|}{ Abastecimento público } & \multicolumn{1}{c|}{ Irrigação } \\
\hline UTD1 - Bacia Metropolitana & UTD2 - Bacia Metropolitana & UTD3 - Bacia Metropolitana \\
\hline UTD4 - Bacia do Curu & UTD5 - Bacia do Curu & UTD6 - Bacia do Curu \\
\hline UTD7 - Bacia do Alto Jaguaribe & UTD8 - Bacia do Alto Jaguaribe & UTD9 - Bacia do Alto Jaguaribe \\
\hline UTD10 - Bacia do Médio Jaguaribe & UTD11 - Bacia do Médio Jaguaribe & UTD12 - Bacia do Médio Jaguaribe \\
\hline UTD13 - Bacia do Baixo Jaguaribe & UTD14 - Bacia do Baixo Jaguaribe & UTD15 - Bacia do Baixo Jaguaribe \\
\hline UTD16 - Bacia do Salgado & UTD17 - Bacia do Salgado & UTD18 - Bacia do Salgado \\
\hline UTD19 - Bacia do Litoral & UTD20 - Bacia do Litoral & UTD21 - Bacia do Litoral \\
\hline UTD22 - Bacia do Acaraú & UTD23 - Bacia do Acaraú & UTD24 - Bacia do Acaraú \\
\hline UTD25 - Bacia do Coreaú & UTD26 - Bacia do Coreaú & UTD27 - Bacia do Coreaú \\
\hline UTD28 - Bacia do Parnaíba & UTD29 - Bacia do Parnaíba & UTD30 - Bacia do Parnaíba \\
\hline UTD31 - Bacia do Banabuiú & UTD32 - Bacia do Banabuiú & UTD33 - Bacia do Banabuiú \\
\hline
\end{tabular}


Os dados referentes às demandas hídricas das principais categorias de uso (indústria, abastecimento público e irrigação) e a disponibilidade hídrica por bacia hidrográfica foi obtido em Ceará (2008).

Todos os fatores de avaliaçáo (insumos e produtos) considerados para avaliar o instrumento da cobrança pela água bruta são listados no Quadro 2. Então, ao todo são sete insumos e dois produtos.

\section{RESULTADOS E DISCUSSÃO}

\section{Seleção de variáveis}

É importante ressaltar que o número de UTDs deve ser grande o suficiente quando comparada com a quantidade de insumos e produtos. Conforme Ferreira e Gomes (2009), uma recomendação prática, em trabalhos recentes, é que o número de UTDs avaliadas seja no mínimo quatro a cinco vezes o número de fatores de avaliação (insumos + produtos).

Com os noves fatores propostos é sugerido que sejam avaliados pelos menos entre 36 e 45 unidades. Contudo, o conjunto das 33 UTDs não pode ser aumentado, então, a única opção é restringir o número de fatores que irão compor o modelo.

Assim, foi usado um método para selecionar os fatores mais significativos de modo a se fazer uma análise mais próxima da realidade. Segundo Meza et al. (2007), os métodos para selecionar os fatores mais representativos devem ser vistos como ferramentas de auxílio à decisão, que apresentam capacidade de orientar à escolha final.
Foi aplicado o método I-O stepwise exaustivo completo para reduzir o número de fatores de avaliação. Para Senra et al. (2007), este método parte do pressuposto que a seleçáo dos fatores deve obedecer ao princípio da máxima relaçáo causal entre insumos e produtos.

Vale salientar que todos os cálculos das eficiências relativas das UTDs desta pesquisa foram realizados com auxílio da ferramenta computacional SIAD (Sistema Integrado de Apoio à Decisão), descrito por Angulo Meza et al. (2005).

O objetivo do método é o de aumentar a eficiência média do conjunto de UTDs com um número limitado de fatores. $\mathrm{O}$ método envolve os seguintes passos:

1. Para cada par insumo/produto, calcula-se a eficiência média do conjunto de UTDs analisadas;

2. O par insumo/produto que obteve a maior eficiência média para o conjunto de UTDs é então escolhido;

3. Ao par escolhido se acrescenta mais um fator e em seguida roda-se o modelo, calculando a eficiência média das UTDs;

4. Escolhe-se para entrar no modelo o fator que gerar a maior eficiência média das UTDs;

5. Se o aumento da eficiência for significativo, acrescenta-se mais um fator ao modelo, repetindo assim o passo 4. Em caso contrário, retira-se o último fator e encerra-se o processo.

QUADRO 2

Fatores avaliação propostos

\begin{tabular}{|c|c|}
\hline \multicolumn{2}{|c|}{ FATORES DE AVALIAÇÃO } \\
\hline Insumos & Produtos \\
\hline $\begin{array}{l}\text { - Número total de usuários cadastrados (NTC); } \\
\text { - Número de usuários faturados (NUF); } \\
\text { - Número de usuários outorgados dentre os faturados } \\
\text { (NUO); } \\
\text { - Volume consumido total durante o período, em m3/ano, } \\
\text { (VCT); } \\
\text { - Volume outorgado total ao ano, em m3/ano, (VOT); } \\
\text { - Demanda hídrica anual, em m3/ano, (DEH); } \\
\text { - Disponibilidade hídrica anual, em m3/ano, (DIH). }\end{array}$ & $\begin{array}{l}\text { - Cobrança faturada total durante o período, em } \mathrm{R} \$ \text { /ano, } \\
\text { (CFT); } \\
\text { - Cobrança arrecadada total durante o período, em } \mathrm{R} \$ / \mathrm{ano} \text {, } \\
\text { (CAT). }\end{array}$ \\
\hline
\end{tabular}


Por intermédio da Figura 3, podem-se mostrar as etapas envolvidas no método I-O stepwise exaustivo completo. Vale salientar que depois de encerrado o processo tem-se um conjunto de fatores que definirá o modelo final de avaliaçáo do conjunto de UTDs proposto.

É importante ressaltar que o tomador de decisão tem toda liberdade para determinar o momento em que o algoritmo deve ser encerrado.Como os fatores definidos no Quadro 2 apresentam tamanhos variados para o conjunto de UTDs, entáo, optou-se por usar a metodologia de análise envoltória de dados com retornos variáveis de escala, ou seja o modelo BCC.

Assim, em cada etapa do método I-O stepwise exaustivo completo foi usado o modelo AED-BCC, com uma orientação a produto. Em seguida, na

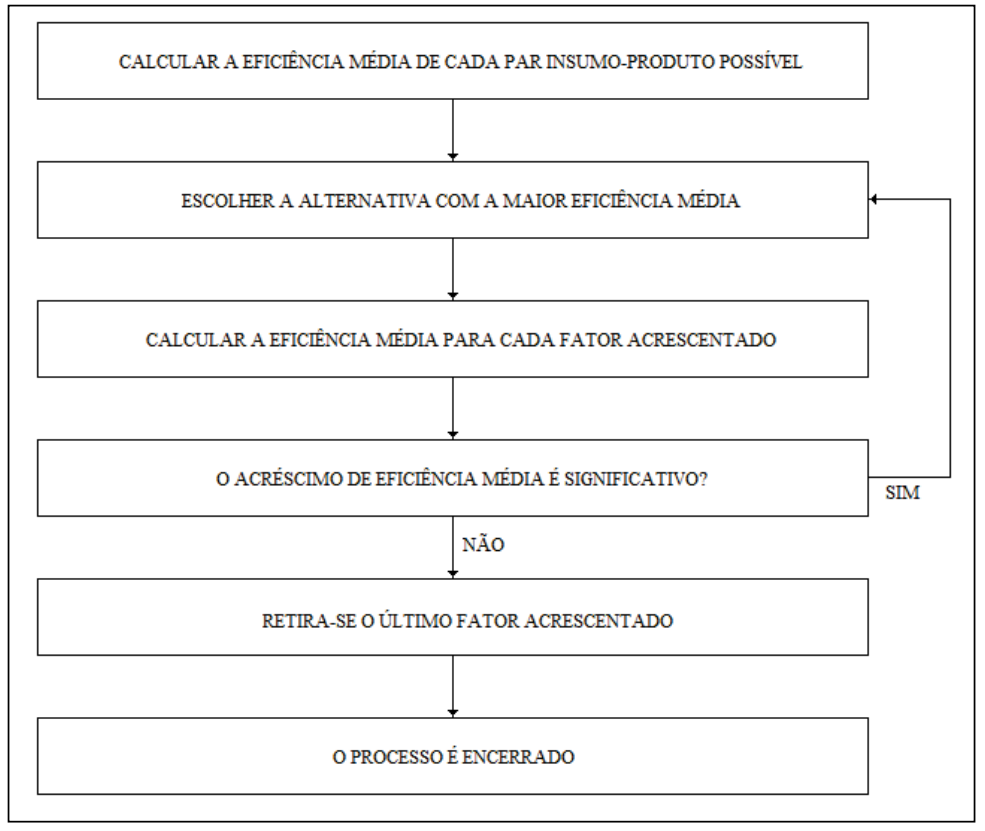

Descrição de cada etapa da aplicação do método l-0 stepwise exaustivo completo

\begin{tabular}{|c|l|c|c|}
\hline Etapa & Conjunto de Fatores & $\begin{array}{c}\text { No UTDs } \\
\text { eficientes }\end{array}$ & Eficiência média \\
\hline I & DEH (I); CFT (P) & 4 & 0,3096 \\
\hline II & NUF (I); DEH (I); CFT (P) & 5 & 0,4775 \\
\hline III & NUF (I); VCT (I); DEH (I); CFT (P) & 9 & 0,5048 \\
\hline IV & NUF (I); VCT (I); DEH (I); DIH (I); CFT (P) & 10 & 0,5438 \\
\hline V & NUF (I); VCT (I); VOT (I); DEH (I); DIH (I); CFT (P) & 11 & 0,5620 \\
\hline VI & NUF (I); NUO (I); VCT (I); VOT (I); DEH (I); DIH (I); CFT (P) & 0,5667 \\
\hline
\end{tabular}

$(I)=$ insumo; $(P)=$ produto
FIGURA 3. Fluxograma das etapas do método $\mathrm{I}-\mathrm{O}$ exaustivo completo.

\section{TABELA 1}


Tabela 1 está ilustrada cada etapa do método I-O, apresentando o conjunto de fatores, o número de UTDs eficientes e a eficiência média do conjunto de unidades.

O ganho de eficiência entre as Etapas V e VI é igual a 0,0047 , o que representa aproximadamente $0,8 \%$ da eficiência média da Etapa V (igual a 0,5620). O acréscimo de eficiência do conjunto entre essas etapas pode ser considerado insignificante, entáo, conforme ilustrado na Figura 3, o fator NUO (número de usuários outorgados dentre os faturados) é retirado do modelo e encerra-se o processo na Etapa V (destacada em vermelho na Tabela 1 ).

Assim, o modelo final, usado para medir a eficiência do conjunto de UTDs, está descrito no Quadro 3. Ou seja, esse modelo apresenta ao todo seis fatores, sendo cinco insumos e um único produto.

\section{QUADRO 3}

Fatores selecionados pelo método l-0 stepwise exaustivo completo

\begin{tabular}{|c|c|}
\hline \multicolumn{2}{|c|}{ FATORES DE AVALIAÇÃO } \\
\hline Insumos & Produtos \\
\hline $\begin{array}{l}\text { - Número de usuários faturados } \\
\text { (NUF); } \\
\text { - Volume consumido total } \\
\text { durante o período, em m3/ } \\
\text { ano, (VCT); } \\
\text { - Volume outorgado total ao } \\
\text { ano, em m3/ano, (VOT); } \\
\text { - Demanda hídrica anual, em } \\
\text { m3/ano, (DEH); } \\
\text { - Disponibilidade hídrica anual, } \\
\text { em m3/ano, (DIH). }\end{array}$ & $\begin{array}{l}\text { - Cobrança faturada } \\
\text { total durante o } \\
\text { período, em } \mathrm{R} \$ \text { /ano, } \\
\text { (CFT); }\end{array}$ \\
\hline
\end{tabular}

\section{Análise da eficiência}

As medidas das eficiências relativas do conjunto analisado, por meio da aplicação do modelo AED-BCC, com orientação a produto, são apresentadas na Tabela 2. Além das medidas de eficiência, a tabela também apresenta os valores dos fatores de avaliação (selecionados pelo método I-O) para cada UTD. É possívelobservar que 11 UTDs são classificadas como eficientes, enquanto as demais 22 UTDs são classificadas como ineficientes. $\mathrm{O}$ conjunto todo de unidades avaliadas nesta pesquisa se apresenta com uma medida de eficiência relativa média igual a aproximadamente $56,1 \%$.
Das 11 unidades eficientes, nove são referentes à cobrança no setor industrial, enquanto uma é do abastecimento e outra da irrigaçáo, conforme descritas no Quadro 4. Ou seja, excetuando as cobranças do abastecimento público, da bacia Metropolitana, e da irrigação, da bacia do Litoral, as demais cobranças eficientes pertencem ao setor da indústria.

QUADRO 4

Descrição de cada unidade classificada como eficiente

\begin{tabular}{|l|l|l|}
\hline \multicolumn{3}{|l|}{ Unidades tomadoras de decisão (UTDs) eficientes } \\
\hline $\begin{array}{l}\text { UTD1 - Indústria } \\
\text { (Metropolitana) }\end{array}$ & $\begin{array}{l}\text { UTD13 - Indústria } \\
\text { (Baixo Jaguaribe) }\end{array}$ & $\begin{array}{l}\text { UTD28 - Indústria } \\
\text { (Parnaíba) }\end{array}$ \\
\hline $\begin{array}{l}\text { UTD2 - Abasteci- } \\
\text { mento (Metropo- } \\
\text { litana) }\end{array}$ & $\begin{array}{l}\text { UTD19 - Indústria } \\
\text { (Litoral) }\end{array}$ & $\begin{array}{l}\text { UTD30 - Irrigação } \\
\text { (Parnaíba) }\end{array}$ \\
\hline $\begin{array}{l}\text { UTD7 - Indústria } \\
\text { (Alto Jaguaribe) }\end{array}$ & $\begin{array}{l}\text { UTD22 - Indústria } \\
\text { (Acaraú) }\end{array}$ & $\begin{array}{l}\text { UTD31 - Indústria } \\
\text { (Banabuiú) }\end{array}$ \\
\hline $\begin{array}{l}\text { UTD10 - Indústria } \\
\text { (Médio Jaguaribe) }\end{array}$ & $\begin{array}{l}\text { UTD25 - Indústria } \\
\text { (Coreaú) }\end{array}$ & \\
\hline
\end{tabular}

Conforme ilustrado no Quadro 4, a cobrança do setor industrial se destaca em relaçáo às demais categorias de uso consideradas neste trabalho. Se forem consideradas somente as unidades desse setor, a cobrança pelo uso da água bruta se apresenta com uma eficiência relativa média de aproximadamente $92,6 \% . N a$ Figura 4, podem ser vista todas as unidades da indústria analisadas, com as suasrespectivas medidas de eficiências relativas.

É importante ressaltar que a indústria é o setor que apresenta as maiores tarifas de cobrança de água, sendo então uma categoria muito representativa em relaçáo ao faturamento e à arrecadação com a cobrança pelo uso da água bruta, nas bacias hidrográficas cearenses.

Como mostrado anteriormente apenas à cobrança na bacia Metropolitana, identificada como UTD2, foi classificada como $100 \%$ eficiente para o setor do abastecimento público. As unidades desse setor apresentam medidas de eficiência baixas, como mostrado na Figura 5. Se forem consideradas somente as unidades desse setor, a cobrança pela água bruta apresenta uma eficiência relativa média de aproximadamente $45,9 \%$. 
Rodrigues, M. V. S.; Aquino, M. D. de; Thomaz, A. C. F. ¿ Análise por envoltória de dados utilizada para medir o desempenho relativo...

TABELA 2

Medida da eficiência relativa e os fatores de avaliação

\begin{tabular}{|c|c|c|c|c|c|c|c|}
\hline UTD & NUF & VCT (mªno) & VOT (mªno) & $\mathrm{DEH}\left(\mathrm{m}^{3} / \mathrm{s}\right)$ & $\mathrm{DIH}\left(\mathrm{m}^{3} / \mathrm{s}\right)$ & CFT (R\$/ano) & Eficiência \\
\hline UTD1 & 121 & $21.201 .494,13$ & $55.789 .465,63$ & 7,99 & 20,62 & $29.866 .171,45$ & $100,0 \%$ \\
\hline UTD2 & 42 & $281.970 .523,29$ & $168.885 .538,17$ & 7,48 & 20,62 & $28.223 .806,33$ & $100,0 \%$ \\
\hline UTD3 & 48 & $17.302 .943,33$ & $3.875 .277,43$ & 0,74 & 20,62 & $175.603,94$ & $7,6 \%$ \\
\hline UTD4 & 40 & $156.165,73$ & $17.395,90$ & 0,23 & 11,82 & $67.395,03$ & $73,2 \%$ \\
\hline UTD5 & 14 & $9.038 .515,89$ & $8.400 .609,74$ & 0,31 & 11,82 & $296.192,18$ & $25,5 \%$ \\
\hline UTD6 & 25 & $22.654 .909,29$ & $20.837 .779,08$ & 2,63 & 11,82 & $44.633,69$ & $0,6 \%$ \\
\hline UTD7 & 19 & $25.577,26$ & $56.272,05$ & 0,11 & 21,74 & $11.038,14$ & $100,0 \%$ \\
\hline UTD8 & 21 & $15.021 .544,23$ & $66.858 .257,97$ & 0,35 & 21,74 & $492.255,98$ & $36,0 \%$ \\
\hline UTD9 & 25 & $2.655 .018,85$ & $2.242 .953,73$ & 0,45 & 21,74 & $10.248,18$ & $0,7 \%$ \\
\hline UTD10 & 26 & $640.746,23$ & 0,00 & 0,05 & 31,88 & $276.520,40$ & $100,0 \%$ \\
\hline UTD11 & 17 & $13.583 .183,74$ & $2.160 .509,40$ & 0,13 & 31,88 & $445.120,96$ & $86,8 \%$ \\
\hline UTD12 & 73 & $149.909 .007,51$ & $9.281 .166,47$ & 4,90 & 31,88 & $889.856,13$ & $17,1 \%$ \\
\hline UTD13 & 87 & $149.486,77$ & $29.064,50$ & 0,21 & 1,55 & $64.512,40$ & $100,0 \%$ \\
\hline UTD14 & 7 & $5.226 .680,00$ & $7.878 .171,11$ & 0,23 & 1,55 & $171.278,31$ & $23,7 \%$ \\
\hline UTD15 & 12 & $21.055 .233,12$ & $5.285 .521,51$ & 1,72 & 1,55 & $43.252,74$ & $4,8 \%$ \\
\hline UTD16 & 60 & $583.954,33$ & $1.153 .575,20$ & 0,15 & 6,44 & $252.011,30$ & $45,4 \%$ \\
\hline UTD17 & 32 & $48.047 .543,68$ & $22.256 .012,08$ & 0,85 & 6,44 & $1.574 .518,07$ & $49,4 \%$ \\
\hline UTD18 & 12 & $8.932 .232,08$ & $2.642 .256,49$ & 2,53 & 6,44 & $16.618,23$ & $1,0 \%$ \\
\hline UTD19 & 3 & $205.910,00$ & $150.211,53$ & 0,15 & 1,15 & $302.187,35$ & $100,0 \%$ \\
\hline UTD20 & 6 & $6.545 .175,87$ & $2.369 .032,50$ & 0,20 & 1,15 & $214.485,37$ & $71,0 \%$ \\
\hline UTD21 & 3 & $513.646,44$ & $218.111,74$ & 0,02 & 1,15 & 410,28 & $99,8 \%$ \\
\hline UTD22 & 53 & $681.287,10$ & $919.434,50$ & 0,06 & 12,39 & $294.611,26$ & $100,0 \%$ \\
\hline UTD23 & 35 & $39.020 .086,73$ & $37.092 .185,73$ & 1,10 & 12,39 & $1.278 .688,19$ & $30,6 \%$ \\
\hline UTD24 & 10 & $934.976,11$ & $477.057,71$ & 6,21 & 12,39 & $1.095,52$ & $0,2 \%$ \\
\hline UTD25 & 12 & $75.389,47$ & 0,00 & 0,02 & 3,22 & $32.535,06$ & $100,0 \%$ \\
\hline UTD26 & 6 & $4.283 .941,04$ & $3.459 .543,00$ & 0,50 & 3,22 & $140.384,72$ & $10,9 \%$ \\
\hline UTD27 & 1 & $49.027,00$ & $2.075 .700,63$ & 0,09 & 3,22 & 23,83 & $96,1 \%$ \\
\hline UTD28 & 13 & $89.622,00$ & $96.177,50$ & 0,01 & 6,94 & $38.677,26$ & $100,0 \%$ \\
\hline UTD29 & 11 & $16.190 .356,62$ & $7.029 .251,10$ & 0,52 & 6,94 & $530.558,01$ & $29,3 \%$ \\
\hline UTD30 & 26 & $9.567 .450,32$ & $5.202 .867,89$ & 0,00 & 6,94 & $16.582,35$ & $100,0 \%$ \\
\hline UTD31 & 11 & $25.305,22$ & 0,00 & 0,23 & 21,66 & $10.920,71$ & $100,0 \%$ \\
\hline UTD32 & 13 & $15.052 .458,03$ & 13.410.396,71 & 0,32 & 21,66 & $493.269,09$ & $41,9 \%$ \\
\hline UTD33 & 87 & $15.407 .150,77$ & $6.549 .775,50$ & 8,75 & 21,66 & $26.996,31$ & $0,7 \%$ \\
\hline
\end{tabular}


Assim, o setor do abastecimento público pode ser considerado como ineficiente em relação à indústria no que diz respeito ao instrumento de cobrança pelo uso da água bruta. Destaca-se negativamente, a bacia do Coreaú, identificada como UTD26, onde a cobrança desse setor apresentou uma eficiência relativa de $10,9 \%$.

Vale lembrar que o abastecimento público em todo o Estado é um setor muito importante, tanto em relação ao consumo de água como em relação ao faturamento e arrecadação com a cobrança pela água bruta. Este setor apresenta tarifas inferiores ao setor da indústria, porém são elevadas quando comparadas as tarifasdo setor da irrigação.

Por fim, o setor considerado mais preocupante foi à irrigação, que se apresentou como o setor mais ineficiente em todo o Estado em relaçáo ao instrumento da cobrança, conforme ilustrado na Figura 6. Apenas a cobrança da irrigação na bacia do Parnaíba, identificada como UTD30, foi classificada como 100\% eficiente. Porém, pode-se considerar a cobrança na bacia do Litoral, identificada como UTD21, como quase eficiente, uma vez que apresentou uma medida de eficiência relativa de 99,8\%.

Se forem consideradas somente as unidades da irrigação, a cobrança pela água bruta desse setor se apresenta em todo o Estado com uma eficiência relativa média de aproximadamente $29,9 \%$, sendo, portanto, considerada como bastante ineficiente quando se comparada com os outros setores da pesquisa. Destaca-se negativamente a cobrança na bacia do Acaraú, identificada como UTD24, com uma medida de eficiência de $0,2 \%$, sendo a unidade mais ineficiente das 33 UTDs analisadas na pesquisa.

A irrigação é o setor que apresenta o maior consumo de água bruta, dentre todas as categorias de uso de recursos hídricos no Estado. Entretanto, é um setor que apresenta baixos faturamentos (ou arrecadação) com a cobrança, uma vez que apresenta as menores tarifas de consumo de água bruta em todo o Estado.
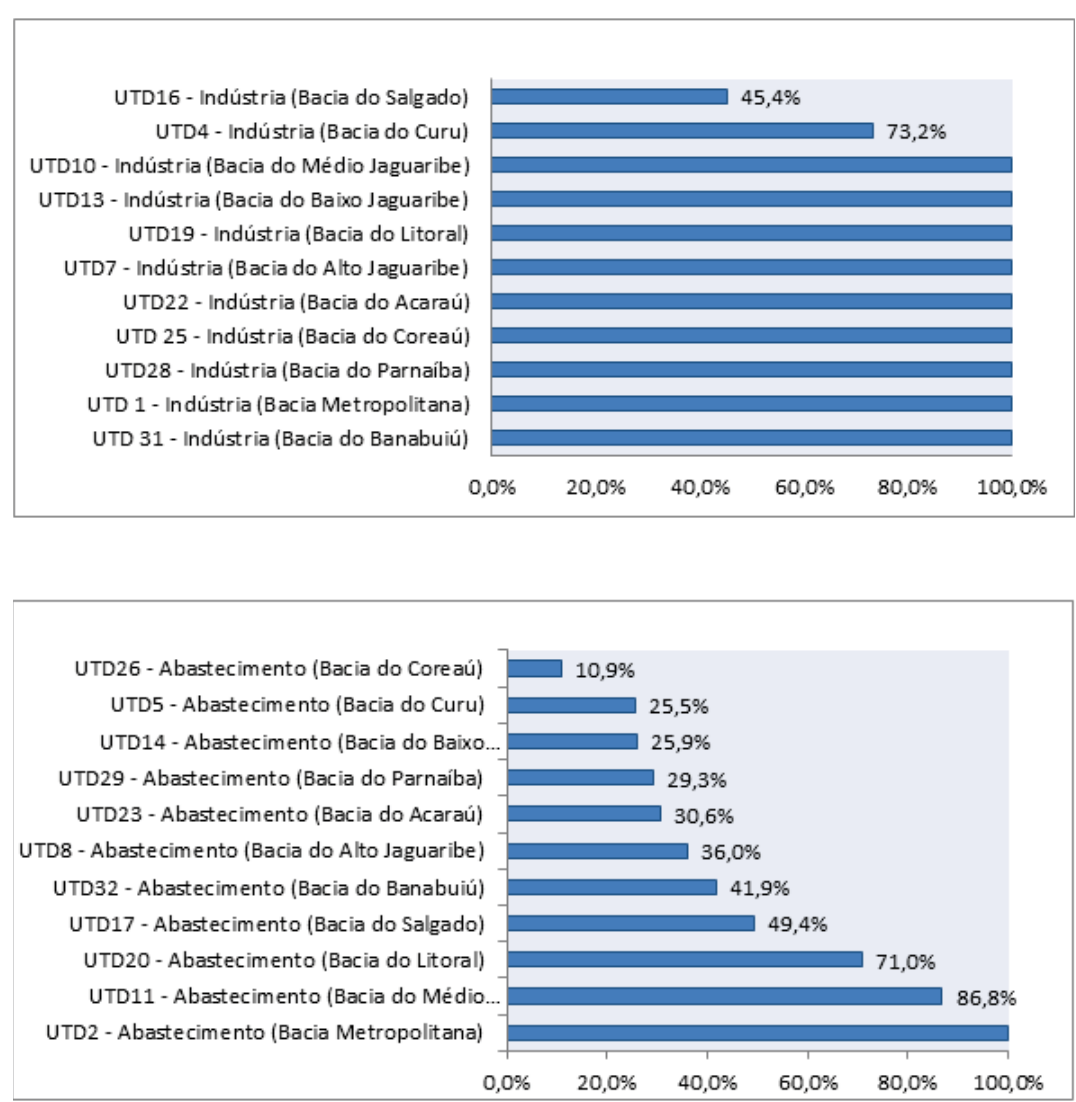

FIGURA 4. Medidas da eficiência relativa das UTDs da indústria.
FIGURA 5. Medidas da eficiência relativa das UTDs do abastecimento público. 
Conforme os resultados obtidos na análise AED realizada nas 33 UTDs, pode-se considerar o setor da indústria bem eficiente, enquanto os setores do abastecimento público e da irrigação são considerados com eficiências muito baixas. Ou seja, as UTDs da indústria são as unidades que melhores estão utilizando os seus recursos para gerar o produto (o faturamento com a cobrança).

Em resumo, pode-se considerar que o modelo deavaliação sugerido na pesquisa se mostrou bastante eficaz em seu propósito, ou seja, o mesmo apresentouum diagnóstico do instrumento da cobrança em todo o Estado, por meio da análise de eficiência das principais categorias de uso da água nas bacias cearense.

Os autores recomendam fortemente o uso desta metodologia científica como apoio no processo de tomadas de decisóes no setor de gerenciamento de recursos hídricos para o cálculo de indicadores de desempenho do instrumento da cobrança, bem como qualquer outro instrumento de políticas públicas.

\section{CONCLUSÕES}

Dos nove fatores proposto para a avaliaçáo, apenas seis foram selecionados por meio do método I-O stepwise exaustivo completo, sendo cinco insumos e um produto (ver Quadro 3). Em seguida, a aplicação do modelo AED-BCC, orientado a produto, no conjunto das 33 UTDs obteve o seguinte resultado: 11 UTDs classificadas como eficientes, sendo $9 \mathrm{da}$ indústria, enquanto 22 UTDs classificadas como ineficientes, sendo a maioria do abastecimento e da irrigação.A cobrança pelo uso da água em todo o Estado se apresentou com uma eficiência relativa média de aproximadamente $56,2 \%$.

Em relação à categoria de uso, a cobrança do setor da indústria se apresenta com a maior eficiência relativa média (aproximadamente 92,6\%), enquanto as cobranças do abastecimento e da irrigação se apresentam com eficiências relativas médias muito inferiores à indústria, com medidas aproximadas a $46,1 \%$ e $29,9 \%$, respectivamente.

Este resultado enfatiza a importância do setor da indústria em relação ao instrumento de cobrança em todo o Estado do Ceará. Além de ser um setor que apresenta um grande volume consumido de água bruta e de arrecadação com a cobrança, a indústria se apresenta como o setor de maior eficiência quando comparada aos demais setores considerados na pesquisa.

As categorias do abastecimento público e da irrigaçáo são setores considerados estratégicos para o Estado em relação à conservação dos recursos hídricos, uma vez queos mesmos são os setores que apresentam os maiores consumos dentre as demais categorias. Entretanto, essas duas categorias se apresentaram com medidas deeficiência abaixo da eficiência média de todo o Estado.

A irrigação pode ser considerada como o setor mais preocupante na análise feita, uma vez que suas unidades apresentaram as menores medidas de eficiência de todo o conjunto, tendo como destaque negativo a cobrança desse setor na bacia do Acaraú, que apresentou uma medida de eficiência quase nula.

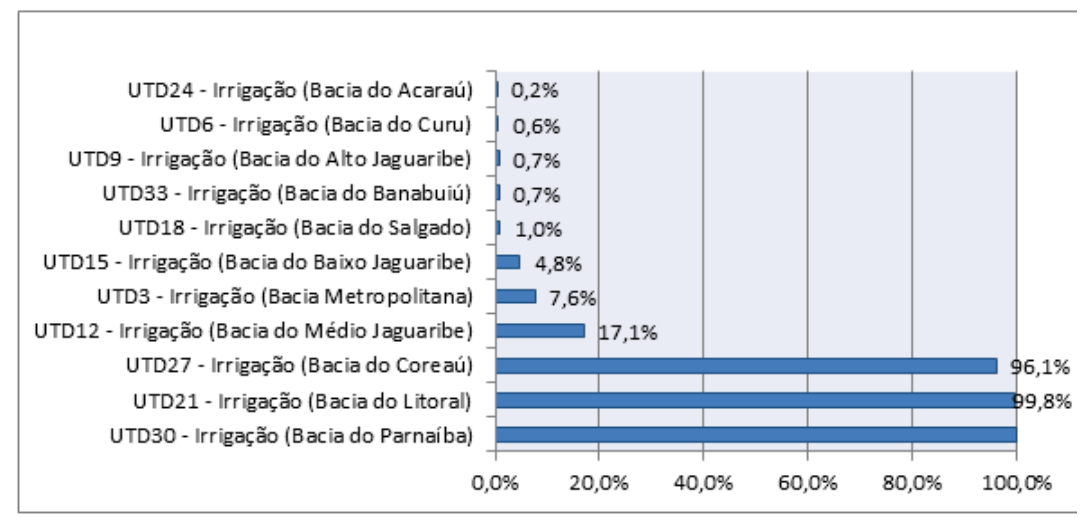

FIGURA 6. Medidas da eficiência relativa das UTDs da irrigação 


\section{Referências}

ADLER, N.; YAZHEMSKY, E. Improving discrimination in data envelopment analysis: PCA-DEA or variable reduction. European Journal of Operational Research, 202, p. 273-284, 2010.

ALI, A. I.; LERME, C. S.; SEIFORD, L. M. Components of efficiency evaluation in Data Envelopment Analysis. European Journal of Operational Research, v. 80, p. 462-473, 1995.

ANGULO MEZA, L.; BIONDI NETO, L.; SOARES DE MELLO, J. C. C. B.; GOMES, L. G. ISYDS - Integrated System for Decision Support (SIAD - Sistema Integrado de Apoio à Decisão): A software package for data envelopment analysis model. Pesquisa Operacional, v. 25 , n. 3, p. 493-503, 2005.

AQUINO, T. S. de A.; GOMES, C. C.; SOUZA FILHO F. de A. de; SILVA, S. M. O. da. Impacto da recuperação do investimento em infraestrutura hídrica na cobrança pelo uso da água.Revista Brasileira de Recursos Hídricos (RBRH), v. 18, n. 1, p. 87-98, 2013.

ATHANASSOPOULOS, A. D. Discriminating among relatively efficient units in data envelopment analysis: A comparison of alternative methods and some extensions. American Journal of Operations Research, v. 2, p. 1-9, 2012.

BANAEIAN, N.; OMID, M.; AHMADI, H. Application of data envelopment analysis to evaluate efficiency of commercial greenhouse strawberry. Research Journal of Applied Sciences, Engineering and Technology, v. 3, n. 3, p. 185-193, 2011.

BANKER, R. D. Maximum likelihood, consistency and Data Envelopment Analysis: A statistical foundation. Management Science, v. 39 , n. 10, p. 1265-1273, 1993.

BANKER, R. D.; CHARNES, A.; COOPER, W. W. Some models for estimating technical and scale inefficiencies in Data Envelopment Analysis. Management Science, v. 30, n. 9, p. 1078-1092, 1984.

BARBOSA, R. de P.; BASTOS, A. P. V. Utilização da análise por envoltória de dados (DEA) na mensuração da eficiência das prestadoras de serviços de água e esgotamento sanitário: Um enfoque no desempenho da companhia de saneamento do estado do Pará. Revista Economia e Gestão, v. 14, n. 35, p. 151-181, 2014.

CAMPOS, M. V. C. de V.; RIBEIRO, M. M. R.; VIEIRA, Z. M. de C. L. A gestão de recursos hídricos subsidiada pelo uso de indicadores de sustentabilidade. Revista Brasileira de Recursos Hídricos (RBRH), v. 19, n. 2, p. 209-222, 2014.

CEARÁ. Assembleia Legislativa. Cenário Atual dos Recursos Hídricos do Ceará - Conselho de Altos Estudos e Assuntos Estratégicos, Assembleia Legislativa do Estado do Ceará; Eudoro Walter de Santana (Coordenador) - Fortaleza: INESP, 2008.

CEARÁ. Lei $n^{0}$ 14.844, de 28 de dezembro de 2010. Fortaleza - CE, 2010.

CHARNES, A.; COOPER, W. W.; RHODES, E. Measuring the efficiency of decision making units. European Journal of Operational Research, v. 2, n. 6, p. 429-444, 1978.

EL-MAHGARY, S. Data Envelopment Analysis - A basic glossary. Operation Research Society, v. 8, p. 15-22, 1995.

FERREIRA, C. M. de C.; GOMES, A. P. Introdução à análise envoltória de dados: teoria, modelos e aplicações. Viçosa - MG: Editora UFV, 2009. 389 p.

GOMES, E. G.; MELLO, J. C. C. B. S. de; FREITAS, A. C. R. de. Efficiency measures for a non-homogeneous group of family farmers. Revista Pesquisa Operacional, v. 32, n. 3, p. 561-574, 2012.

GOMES JÚNIOR, J. F.; SOARES DE MELLO, J. C. C. B.; ANGULO MEZA, L. DEA nonradial efficiency based on vector properties. International TransactionsInoperational Research, 20, p. 341-364, 2013.

HAJKOWICZ, S.; COLLINS K. A review of Multiple Criteria Analysis for Water Resource Planning and Management. Water Resour Manage, v. 21, p. 1553-1566, 2007.

MARJANOVIC, D.; SARKOCEVIC, Z.; MISIC, M.; STOJCETOVIC, B. Data envelopment analysis application for assessing the efficacy of MSP. $8^{\text {th }}$ International Quality Conference. Center for Quality, Faculty of Engineering, University of Kragujevac. 2014.

MEZA, L. A.; SOARES DE MELLO, J. C. C. B.; GOMES, E. G.; FERNANDES, A. J. S. Selecção de variáveis em DEA aplicada a uma análise do mercado de energia eléctrica. Investigação Operacional, v. 27, p. 21-36, 2007.

RODRIGUES, M. V. S. Avaliação do desempenho da cobrança da água bruta por categoria de uso nas bacias do Estado do Ceará utilizando a análise por envoltória de dados. 2014. 174 f. Tese (Doutorado em Engenharia Civil) - Universidade Federal do Ceará, Fortaleza - CE, 2014.

RODRIGUES, M. V. S.; AQUINO, M. D. de. Análise comparativa entre a cobrança pelo uso da água bruta do Estado do Ceará com a cobrança aplicada no Estado de São Paulo. Revista de Gestão de Águas da América Latina (REGA), v. 11, n. 2, p. 37-51, 2014. SENRA, L. F.A. de C.;NANCI, L. C.; SOARES DE MELLO, J. C. C. B.;ANGULO MEZA, L. Estudo sobre métodos de seleção de variáveis em DEA. Revisa Pesquisa Operacional, v. 27, n. 2, p. 191-207, 2007.

SILVEIRA, J. Q.; SOARES DE MELLO, J. C. C. B.; MEZA, L. A. Evaluación de la eficiencia de las compañías aéreas brasileñas a través de um modelo híbrido de análisis envolvente de datos (DEA) y programación lineal multiobjetivo. Revista Chilena de Ingeniería, v. 20, n. 3, p. 331-342, 2012.

SOUZA, P. C. T.; WILHELM, V. E. Uma introdução aos modelos DEA de eficiência técnica. Revista Tuiuti: Ciência e Cultura, n. 42, p. $121-139,2009$. 
Rodrigues, M. V. S.; Aquino, M. D. de; Thomaz, A. C. F. A Análise por envoltória de dados utilizada para medir o desempenho relativo...

TREVISAN, M. L.; SILVEIRA, G. L. da; CRUZ, J. C.; CRUZ, R. C. Sensibilidade de fatores para valoração do ambiente com o uso de avaliação multicritério e geoprocessamento digital. Revista Brasileira de Recursos Hídricos (RBRH), v. 16, n. 2, p. 39-48, 2011.

Marcus Vinícius Sousa Rodrigues Professor Adjunto - Departamento de Ciências Exatas, Tecnológicas e Humanas (DCETH). Universidade Federal Rural do Semi-Árido (UFERSA) - Campus Angicos (Angicos - RN). E-mail: marcus@ufersa.edu.br.

Marisete Dantas De Aquino Professora Associada - Departamento de Engenharia Hidráulica e Ambiental (DEHA). Universidade Federal do Ceará (UFC) - Campus do Pici (Fortaleza - CE). E-mail: marisete@ufc.br

Antônio Clécio Fontelles Thomaz Professor Adjunto - Centro de Ciências e Tecnologia. Universidade Estadual do Ceará (UECE) - Campus do Itaperi (Fortaleza-CE). E-mail: clecio@larces.uece.br 
